\title{
Optimization free of algorithm-specific control parameters for Power System Stabilizer tuning
}

\author{
M. Ramirez Gonzalez, F. R. Segundo Sevilla, P. Korba \\ Institute of Energy Systems and Fluid Engineering \\ ZHAW Zurich University of Applied Sciences \\ Winterthur, Switzerland
}

\begin{abstract}
A settings-free and derivative-free optimization technique, called Jaya Optimization Algorithm, is investigated in this paper for the design of simple fuzzy PSSs (FPSSs) in a multimachine power system. Performance of this technique during the optimization process is compared to the one exhibited by a Bat Optimization Algorithm based approach. Considering a set of test cases for illustrative purposes, FPSSs tuned with both of these algorithms are applied to the study system and their performance in damping inter-area and local oscillations is analyzed against some commonly used lead-lag structures, including multi-band PSSs.
\end{abstract}

Keywords- Power system stabilizer; fuzzy logic; parameter tuning; settings-free optimization; Jaya Optimization Algorithm

\section{INTRODUCTION}

Power systems are experiencing dramatic changes due to the rapidly increasing energy demand, trends to intensify the application of renewable energy resources and the deregulation of electricity markets [1]. Besides, with the interconnection of large electrical networks to reduce the reserve energy, electromechanical oscillations that significatively affect the security and stability of power systems and lead to cascade outages may appear [2]. Although conventional power system stabilizers have been widely used for many years to reduce the unwanted effects of these oscillations, the operation and control requirements of modern electrical networks have brought about new challenges that demand the introduction of advanced technologies and methods to effectively deal with these issues [3].

Over the past few decades, various computational intelligence based methods such as artificial neural networks, evolutionary computation, swarm intelligence, artificial immune systems, and fuzzy systems have been proposed for solving different complex problems in many areas of power system engineering [4], [5]. These methods have become increasingly popular due to their relatively simple mathematical structure and the improved solutions they can provide, as compared to traditional techniques. Moreover, to take advantage of the strengths of each single method and eliminate individual weaknesses, hybrid paradigms are proposed [6]. For instance, fuzzy systems can be used to approximate and implement human expert control actions through linguistic terms and fuzzy if-then rules. They are appropriate for dealing with uncertainties and approximate reasoning, especially for complex nonlinear processes where an exact mathematical description of the plant would be difficult to derive [4], [6]. However, despite the outstanding performance reported in many fuzzy logic applications, it is widely recognized that the tuning of fuzzy logic systems represents a critical task in their design. Therefore, they can be combined with a suitable optimization algorithm that provides an optimal solution and solve the controller parameter tuning issue.

Population-based optimization algorithms have become very popular in recent years because they provide a relatively simple process, free of derivatives, to search for optimal solutions even in very complex problems. However, in addition to common parameters such as number of generations and population size, some strategies require their own algorithm-specific control parameters, such as mutation and crossover rate in Genetic algorithm, cognitive/social acceleration factors and inertia weights in Particle swarm optimization, and low/high pulse frequency, initial loudness and pulse rate in Bat optimization, for example. Generally, the correct setting of these additional parameters depends on the particular application and has a great influence on the algorithm performance, leading to increased computational effort or local optima when they are not properly tuned [6], [7].

In this work, fuzzy logic is combined with two recently introduced optimization techniques, such as the Bat Optimization Algorithm (BOA) and the Jaya Optimization Algorithm (JOA), to design power system stabilizers (PSSs) in a sample multimachine power system. While a set of own parameters must be defined in BOA, JOA does not use any algorithm-specific control parameters. Although any of these optimization techniques may be applied to tune fuzzy PSSs, their complexity and their performance in aspects such as speed of convergence, precision and computational effort should also be taken into account for an effective application. For illustrative purposes, system response using BOA and JOA based fuzzy PSSs is compared against the performance provided by multiband, conventional $\Delta \omega$, and conventional $\Delta P_{a}$ PSSs.

\section{PROBLEM FORMULATION}

\section{A. Sample Power System}

In this study, the power system illustrated in Fig. 1 is used as the test system, with generators G1 and G2 forming one area, and G3 and G4 forming another area. These areas are connected together by two transmission lines. Parameters of all generators, speed governors, AVRs, transmission lines and loads can be 
obtained from [8]. Operating conditions are given in the Appendix.

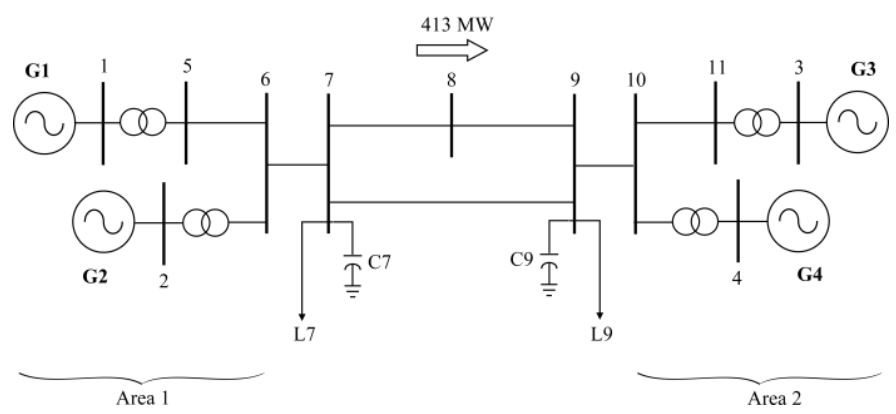

Fig. 1. Sample power system.

With no PSSs, the frequency and damping of the system electromechanical oscillations modes for the base case operating condition are given in Table I, where an inter-area oscillation mode with negative damping can be observed.

TABLE I. ELECTROMECHANICAL OSCILLATION Modes

\begin{tabular}{|c|c|c|}
\hline Mode & Frequency $\mathbf{( H z )}$ & Damping (\%) \\
\hline 1 & 0.64 & -2.6 \\
\hline 2 & 1.12 & 8.0 \\
\hline 3 & 1.16 & 8.0 \\
\hline
\end{tabular}

\section{B. Fuzzy PSS (FPSS)}

To deal with the undamped oscillations of the system, FPSSs with the structure given in [9] will be used in this work. In this regard, the FPSS output $u$ is calculated in the following manner:

$$
u=K_{o} \cdot s_{u} \cdot u_{u s}
$$

where $K_{o}$ is an output scaling factor, $s_{u}$ is the control action sign, and $u_{u s}$ is determined from the fuzzy inference process using the rule base in Table II.

TABLE II. RULE BASE

\begin{tabular}{|c|c|c|c|c|}
\hline $\boldsymbol{d}$ & $Z O$ & $S$ & $M$ & $B$ \\
\hline $\boldsymbol{u}_{\boldsymbol{u} \boldsymbol{s}}$ & $Z O$ & $S$ & $M$ & $B$ \\
\hline
\end{tabular}

In Table II, ZO, S, $M$ and $B$ refer to Zero, Small, Medium and $B i g$, respectively. While these terms are represented by triangular membership functions evenly distributed in the range $\{0,1\}$ for the normalized variable $d$, fuzzy singletons are considered for the ones associated with $u_{u s} . d$ is defined in this case by the following expressions:

$$
\begin{gathered}
y=K_{1} \cdot \Delta \omega+K_{2} \cdot \Delta \dot{\omega} \\
d=|y|
\end{gathered}
$$

where $\Delta \omega$ and $\Delta \dot{\omega}$ are the machine speed deviation and its rate of change, respectively, and $K_{1}$ and $K_{2}$ are their associated scaling factors. The sign of the control action is simply computed from:

$$
s_{u}=\left\{\begin{array}{cc}
1 & \text { if } \quad y \geq 0 \\
-1 & \text { otherwise }
\end{array}\right.
$$

\section{Constrained optimization problem}

Parameters of the FPSS, namely, $K_{1}, K_{2}$, the ones related to the triangular membership functions and singletons, and $K_{o}$ are adjustable parameters that may enhance overall system damping. However, $K_{o}$ is normally defined by the physical restrictions associated with the controller output. As for the parameters of triangular membership functions and singletons, they can be adjusted in a relatively simple way by working only on the center points of the representations. Therefore, $K_{1}, K_{2}$ and the parameter that defines the centers of the fuzzy sets $S$ and $M$ are selected to be optimized in this study according to the minimization of the following cost function:

$$
J=\sum_{1}^{D} \sum_{k=1}^{t_{\text {sim }}}\left(c_{1} \cdot \Delta \omega_{k}+c_{2} \cdot \Delta u_{k}\right) \cdot t_{k}
$$

where, $D$ refers to the number of disturbances for parameter optimization, $t_{s i m}$ is the respective simulation time, and $c_{1}$ and $c_{2}$ are weighting factors. Besides, $\Delta \omega_{k}$ and $\Delta u_{k}$ were defined as:

$$
\begin{gathered}
\Delta \omega_{k}=\left|\Delta \omega_{13, k}-\Delta \omega_{13, k-1}\right|+\left|\Delta \omega_{12, k}-\Delta \omega_{12, k-1}\right|+ \\
\left|\Delta \omega_{34, k}-\Delta \omega_{34, k-1}\right| \\
\Delta u_{k}=\left|u_{1, k}-u_{1, k-1}\right|+\left|u_{2, k}-u_{2, k-1}\right|+ \\
\left|u_{3, k}-u_{3, k-1}\right|+\left|u_{4, k}-u_{4, k-1}\right|
\end{gathered}
$$

with $\Delta \omega_{a b, k}$ being the relative speed differences between machines $a$ and $b$, and $u_{n, k}$ referring to the FPSS output at machine $n$. The tuning of FPSSs can then be carried out through the solution of the following constrained optimization problem:

$$
\begin{gathered}
\text { Minimize J subject to: } \\
K_{1}^{\min } \leq K_{1} \leq K_{1}^{\max } \\
K_{12}^{\min } \leq K_{2} \leq K_{2}^{\max } \\
S_{c p}^{\min } \leq S_{c p} \leq S_{c p}^{\max } \\
M_{c p}^{\min } \leq M_{c p} \leq M_{c p}^{\max }
\end{gathered}
$$

\section{CONSIDERED OPTIMIZATION ALGORITHMS}

To solve the problem in (8), BOA and JOA based strategies are used in this work. A brief review of these optimization algorithms is given below.

\section{A. $B O A$}

In the search for better and more efficient algorithms, the bat optimization algorithm was introduced in [10]. It is a bioinspired strategy based on the echolocation behavior of microbats. By associating each member in the bat population with a pulse frequency $f_{i}$, a velocity $v_{i}$ and a location $x_{i}$, the main equations of the algorithm are:

$$
\begin{gathered}
f_{i}=f_{\text {min }}+\left(f_{\text {max }}-f_{\text {min }}\right) \cdot \beta_{i} \\
v_{i}^{t}=v_{i}^{t-1}+\left(x_{i}^{t-1}-x^{*}\right) \cdot f_{i}
\end{gathered}
$$




$$
x_{i}^{t}=x_{i}^{t-1}+v_{i}^{t}
$$

where $f_{\min }$ and $f_{\text {max }}$ are respectively the minimum and maximum values of the pulse frequency, $\beta$ is a randomly selected number in the interval 0 to 1 , and $x *$ is the best solution up to the present time.

To provide a way to automatically switch from explorative shifts to intensive and local exploitation as the search approaches global optimality, the loudness $A$ and pulse rate $r$ can be regulated according to the following expressions:

$$
\begin{gathered}
A_{i}^{t+1}=\alpha A_{i}^{t} \\
r_{i}^{t+1}=r_{i}^{0}[1-\exp (-\gamma t)]
\end{gathered}
$$

with $\alpha$ and $\gamma$ being constants. Detailed information of the algorithm and its diverse applications can be found in [7], [10]. The flowchart of the BOA strategy is shown in Fig. 2.

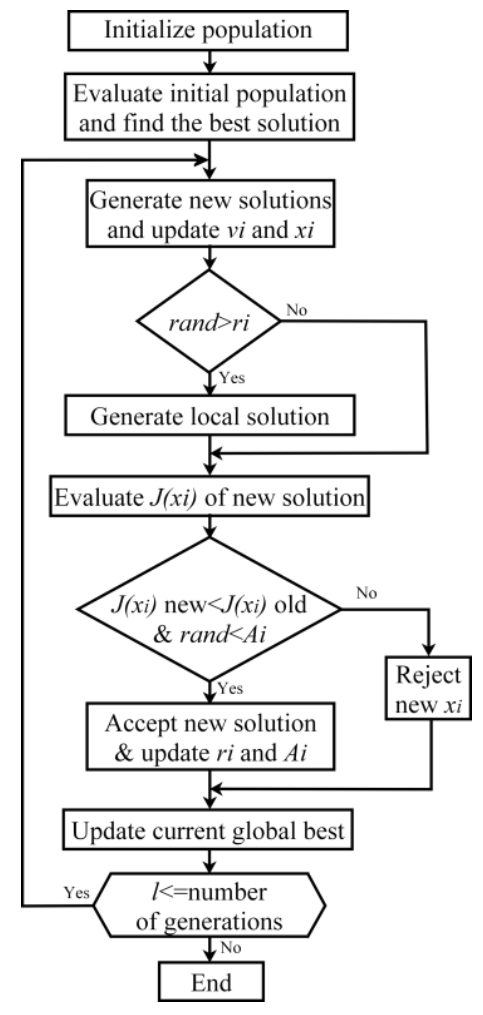

Fig. 2. BOA flowchart.

\section{B. JOA}

JOA is a relatively new optimization technique characterized by no algorithm-specific control parameters, which makes it very simple to implement and apply [11]. In this case, for a candidate solution $x_{i}$ at iteration $t$, the solution will be updated according to the following:

$$
\begin{aligned}
x_{i}^{t}= & x_{i}^{t-1}+\operatorname{rand}_{1}\left(x_{\text {best }}-x_{i}^{t-1}\right) \\
& -\operatorname{rand}_{2}\left(x_{\text {worst }}-x_{i}^{t-1}\right)
\end{aligned}
$$

where $x_{\text {best }}$ and $x_{\text {worst }}$ respectively represent the best and worst solutions up to now, and rand $_{1}$ and rand 2 refer to random numbers in the range 0 to 1 for each search variable. While the second term in (14) represents the tendency of the solution to always try to get closer to the best solution in each generation, the last term in the expression has to do with moving away from the worst solution [11]. As clearly observed from (14), JOA represents an algorithm-specific parameter-less optimization process. A detailed description of this algorithm and its engineering applications can be found in [11], [12]. Fig. 3 illustrates the flowchart of the JOA approach.

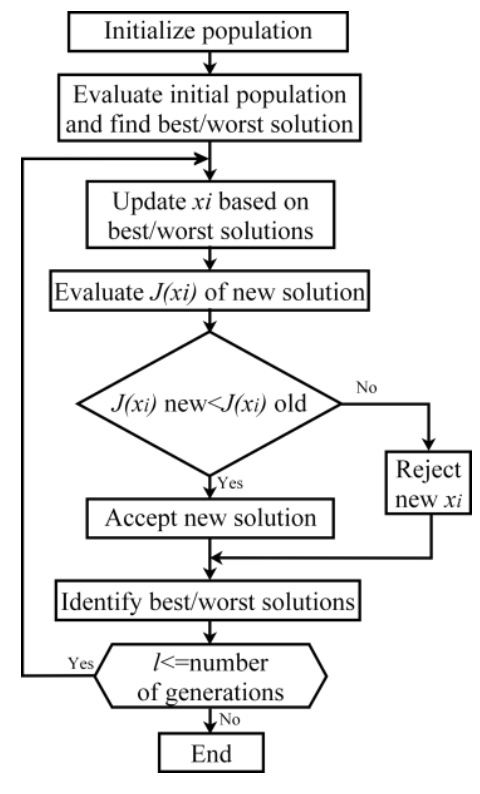

Fig. 3. Flowchart of JOA.

\section{SimUlation RESUlts}

\section{A. Design of fuzzy PSSs}

To enhance power system damping and for the purpose of illustrating the performance of the considered optimization algorithms in this study, the four generators of the test system were equipped with FPSSs. As for the involved optimization strategies, the number of generations and population size were respectively fixed to 100 and 20, and the initial populations were set in a randomly way for both BOA and JOA. Now, for the BOA alternative, suggested parameters of the algorithm, as given in [7], were used in the studies. In this regard, low and high frequency limits were fixed to 0 and 2, respectively, initial loudness was randomly chosen in the range $\{1,2\}$, pulse rate was initially specified as 0.1 , and $\alpha$ and $\gamma$ were settled to 0.9 . On the other hand, JOA did not require any own algorithm-specific control parameters to be set.

Both optimization alternatives were implemented in Matlab, and the study system and FPSSs in Simulink [13], as graphically illustrated in Fig. 4. The parameter tuning process in this work was carried out under the application of different critical system perturbations for the base case operating condition, namely, a three phase fault at bus 7 and 9 without line outage. Nonlinear time domain simulations were carried out to evaluate each candidate solution and compute the cost function value during each iterative evolution process. The best final solutions after ten runs are given in Table III. Convergence results of cost function minimization for the algorithms are illustrated in Fig. 5, and corresponding fitness values achieved during the optimization process are provided in Table IV. 
FPSS

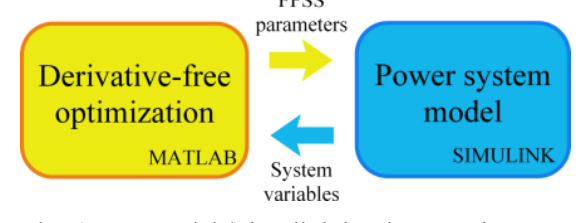

Fig. 4. Matlab/Simulink implementation.

TABLE III. BEST FinAL SOLUTIONS

\begin{tabular}{|c|c|c|c|}
\hline \multirow{3}{*}{ Parameter } & \multirow{2}{*}{ FPSS } & \multicolumn{2}{|c|}{ Algorithm } \\
\cline { 3 - 4 } & & BOA & JOA \\
\hline \multirow{4}{*}{$K_{I}$} & G1 & 290.54 & 294.45 \\
\hline & G2 & 283.59 & 295.81 \\
\hline & G3 & 257.61 & 343.60 \\
\hline & G4 & 220.49 & 301.59 \\
\hline \multirow{4}{*}{$K_{2}$} & G1 & 48.02 & 47.62 \\
\hline & G2 & 51.62 & 52.18 \\
\hline & G3 & 51.60 & 47.82 \\
\hline & G4 & 43.69 & 35.64 \\
\hline \multirow{5}{*}{$S_{c p}$} & G1 & 0.62 & 0.58 \\
\hline & G2 & 0.49 & 0.66 \\
\hline & G3 & 0.22 & 0.45 \\
\hline & G4 & 0.41 & 0.66 \\
\hline \multirow{5}{*}{$M_{c p}$} & G1 & 0.94 & 0.98 \\
\hline & G2 & 0.64 & 0.99 \\
\hline & G3 & 0.73 & 0.79 \\
\hline & G4 & 0.71 & 0.92 \\
\hline
\end{tabular}

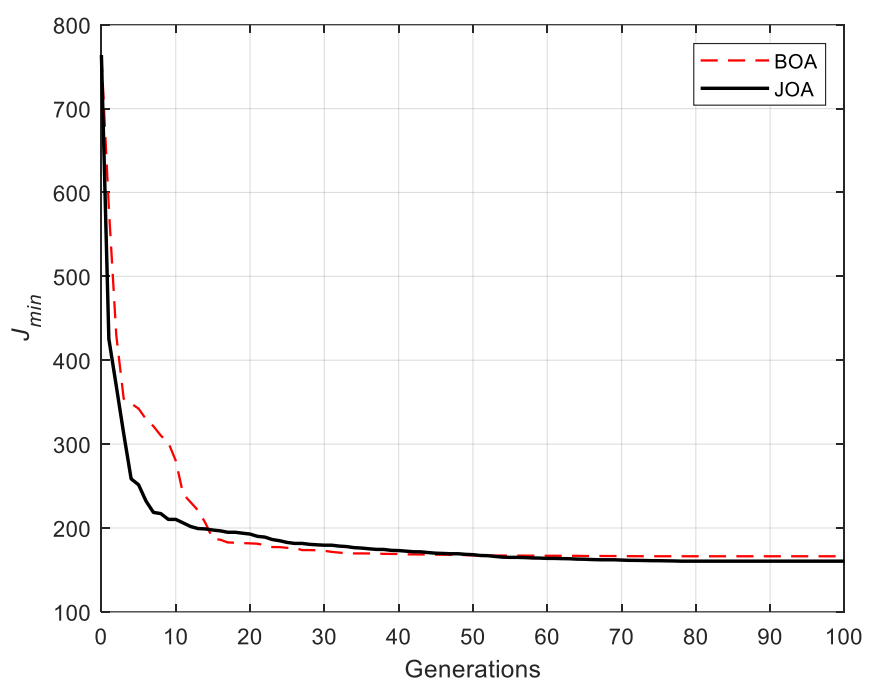

Fig. 5. Cost function convergence averaged over 10 runs.

TABLE IV. FitNESS VALUE DURING OptimizATION

\begin{tabular}{|c|c|c|c|c|}
\hline \multirow{2}{*}{ Algorithm } & \multicolumn{3}{|c|}{$\boldsymbol{J}_{\text {min }}$} & $\begin{array}{c}\text { Average, relative } \\
\text { computational } \\
\text { time (pu) }\end{array}$ \\
\cline { 2 - 5 } & Best & Worst & Mean & 1.00 \\
\hline BOA & 158.98 & 179.88 & 166.24 & 0.92 \\
\hline JOA & 158.59 & 162.87 & 160.42 & \\
\hline
\end{tabular}

From Fig. 5, it can be observed that both BOA and JOA were able to achieve comparable convergence rates and average precision. From Table IV, it is also clear that best minimizing results after ten independent runs are very similar in both approaches for the problem under consideration. As for the computational time, an average $8 \%$ reduction with the JOA alternative can be noticed in this study, as compared to BOA.

\section{B. Test cases and results}

For illustrative purposes, system response under the effect of fuzzy PSSs designed with BOA (BOA-FPSS) and JOA (JOAFPSS) is investigated for the following test cases:

1) A three phase fault is applied at bus 8 in the base case operating condition $\left(\mathrm{OC}_{1}\right)$. Then, it is cleared after 5 cycles by disconnecting the line between buses 7-8 and 8-9.

2) A $5 \%$ step change is applied on the reference voltage of machine $\mathrm{G} 1$ for 12 cycles in $\mathrm{OC}_{1}$.

3) A three phase fault is applied at bus 9 in the operating condition $\mathrm{OC}_{2}$, where active power from Area 1 to Area $2\left(\mathrm{P}_{\mathrm{B} 7}\right.$ B9) has been increased to $487 \mathrm{MW}$. The fault is cleared after 8 cycles with no line outage.

Power system performance for the test cases above and all the perturbations occurring at $1 \mathrm{~s}$ is shown in Figs. 6 through 11. System behavior with multi-band (MB), conventional $\Delta \omega$, and conventional $\Delta P_{a}$ PSSs is also included in the studies for comparison purposes [14]. The center frequencies and corresponding gains of each band in the MB-PSSs were selected according to a desired and nearly flat phase response between $0.1 \mathrm{~Hz}$ and $5 \mathrm{~Hz}$. The settings for the $\Delta \omega$-PSS and $\Delta P_{a}$-PSSs were derived from [8]. Parameters for $\mathrm{MB}, \Delta \omega$ and $\Delta P_{a}$ PSSs, as used in the study, are provided in the Appendix [14].

As can be observed from Fig. 6, the $\Delta P_{a}$-PSSs fails to keep system stability under the large disturbance considered in test case 1 . For this case, although system synchronism is maintained with the rest of PSSs, interarea and local oscillations of the sample system are much better damped with MB-PSSs, BOAFPSSs and JOA-FPSSs, as can be noticed from Figs. 6 and 7.

Now, from the results in Figs. 8 and 9, a superior performance of MB-PSSs, BOA-FPSSs and JOA-FPSSs in damping interarea and local oscillations modes can also be seen under the application of a small disturbance, as compared to $\Delta \omega$ PSSs and $\Delta P_{a}$-PSSs. It can be observed that these two last PSSs show less effectiveness in quickly reaching the steady state of the system after applying a 5\% step change on the reference voltage of generator G1.

System response to a large perturbation considering a somewhat different operating point is illustrated in Figs. 10 and 11. One more time, the system can more effectively reach stable operating conditions with the MB-PSSs, BOA-FPSSs and JOAFPSSs. However, it can be noticed that the performance of the MB-PSSs has been somehow affected with the change in the system operating point since, as compared to the BOA-FPSSs and JOA-FPSSs, a longer time for damping interarea and local oscillations is perceived with this option. 


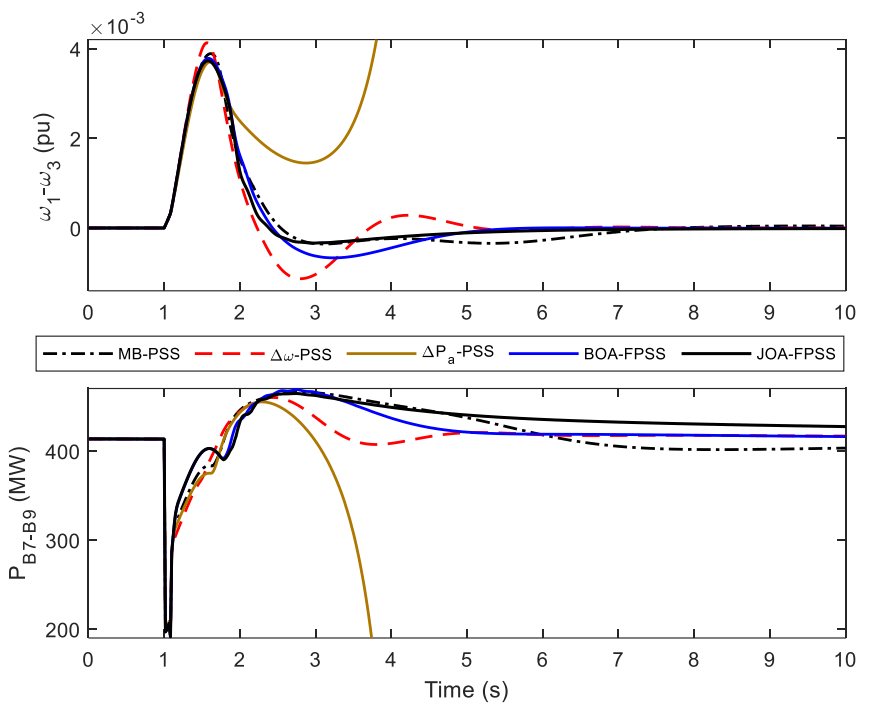

Fig. 6. Simulation results for test case 1: $\omega_{1}-\omega_{3}$ and $\mathrm{P}_{\mathrm{B} 7-\mathrm{B} 9}$ response.
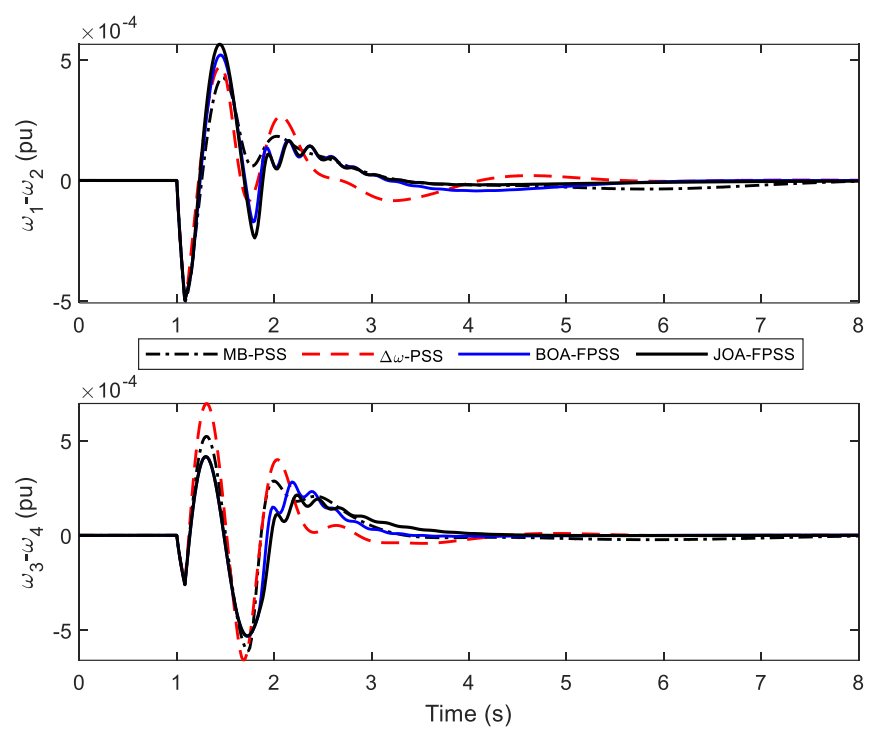

Fig. 7. Simulation results for test case 1: $\omega_{1}-\omega_{2}$ and $\omega_{3}-\omega_{4}$ response.
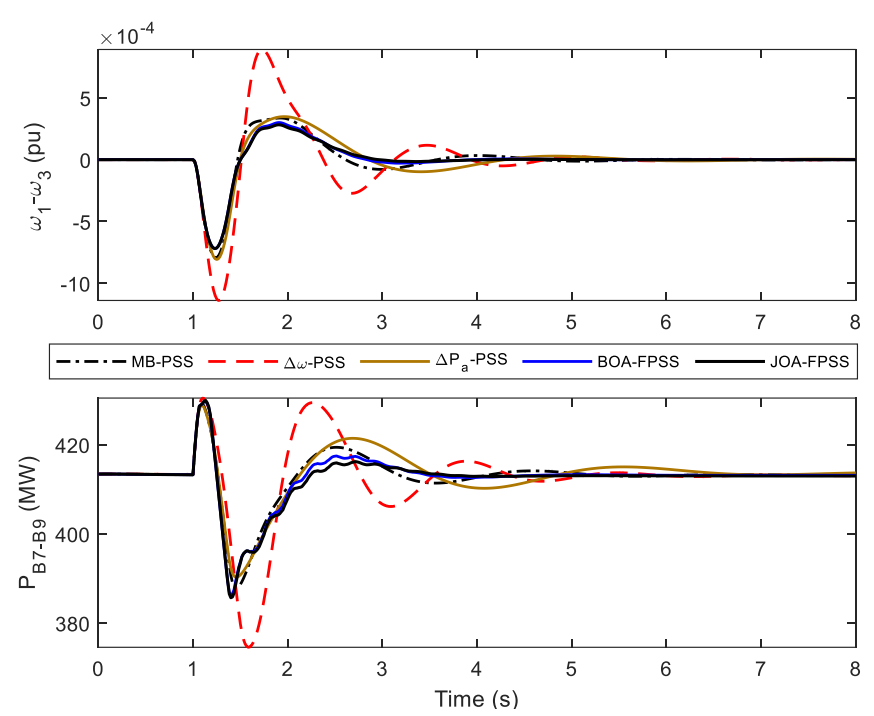

Fig. 8. Simulation results for test case 2: $\omega_{1}-\omega_{3}$ and $\mathrm{P}_{\mathrm{B} 7 \mathrm{~B} 9}$ response.

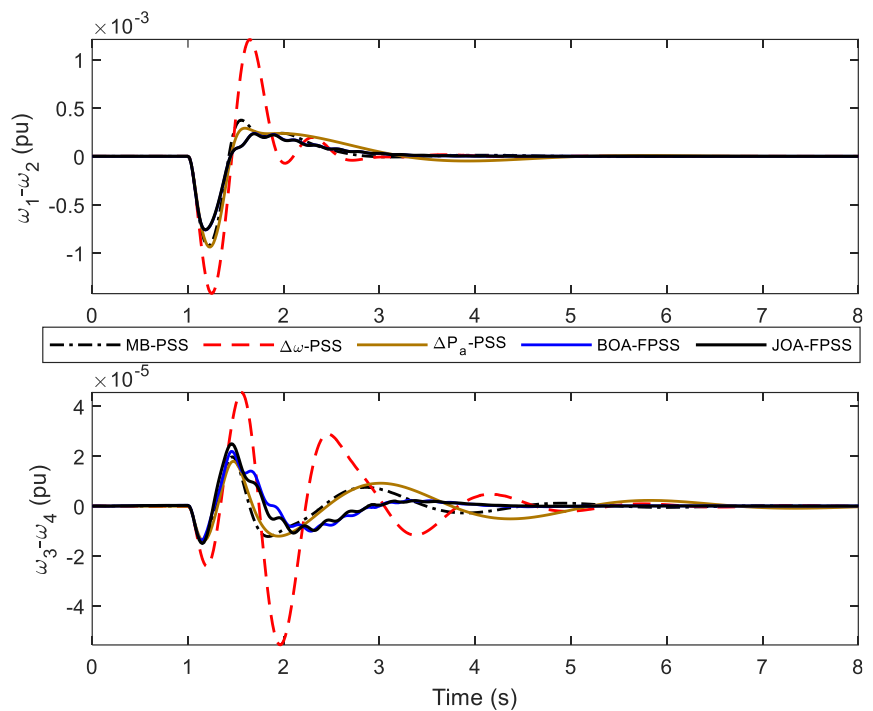

Fig. 9. Simulation results for test case 2: $\omega_{1}-\omega_{2}$ and $\omega_{3}-\omega_{4}$ response.
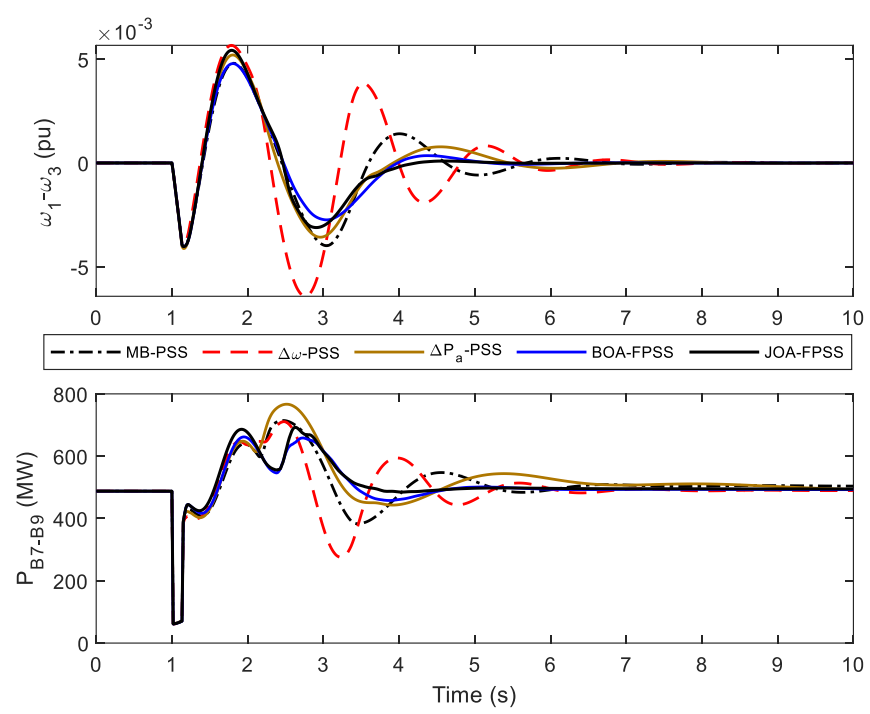

Fig. 10. Simulation results for test case 3: $\omega_{1}-\omega_{3}$ and $\mathrm{P}_{\mathrm{B} 7 \mathrm{~B} 9}$ response.
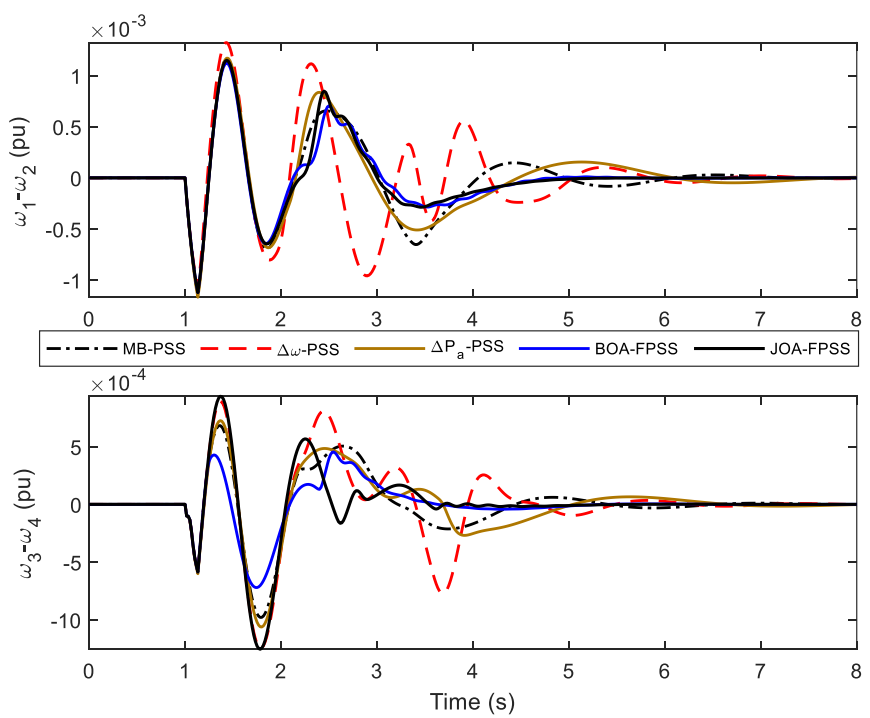

Fig. 11. Simulation results for test case 3: $\omega_{1}-\omega_{2}$ and $\omega_{3}-\omega_{4}$ response 
To quantitatively measure and compare the performance of the different PSSs considered in this study, the computation of the cost function (5) was carried out separately for each test case and is given in Table V. According to this, JOA-FPSSs are able to provide the best performance in Cases 1 and 2, while a relatively better response in these terms is obtained with BOAFSSs for Case 3. On the other hand, a comparatively poor performance is exhibited in general by $\Delta \omega$-PSSs and $\Delta P_{a}$-PSSs in this study. As for MB-PSSs, a remarkable system response can be achieved in Case 1 and a pretty good performance can also be accomplished in Case 2, as observed from the values of $J$ in Table V. However, although the response of MB-PSSs is acceptable in Case 3, a significant difference in terms of $J$ is noticed against the BOA-FPSSs alternative, which provides the minimum value in this case. All these observations coincide with the time domain simulation results previously discussed.

TABLE V. Cost Function VALue For Test CASES

\begin{tabular}{|c|c|c|c|}
\hline \multirow{2}{*}{ PSS } & \multicolumn{3}{|c|}{$\boldsymbol{J}$} \\
\cline { 2 - 4 } & Case 1 & Case 2 & Case 3 \\
\hline MB-PSS & 26.51 & 8.38 & 105.13 \\
\hline$\Delta \omega$-PSS & 34.69 & 19.35 & 184.51 \\
\hline$\Delta P_{a}$-PSS & Unstable & 8.94 & 95.11 \\
\hline BOA-FPSS & 27.50 & 6.43 & 71.20 \\
\hline JOA-FPSS & 24.53 & 6.25 & 78.70 \\
\hline
\end{tabular}

\section{CONCLUSIONS}

Design of simple fuzzy PSSs using two modern and derivative-free optimization algorithms such as BOA and JOA has been carried out in this study. Although both strategies were able to accomplish comparable results during the optimization process, JOA showed on average a lower computational time in this sense. When compared to some commonly used lead-lag PSSs, such as MB-PSSs, $\Delta \omega$-PSSs and $\Delta P_{a}$-PSSs, BOA-FPSSs and JOA-FPSSs succeeded in providing a relatively better damping effect to inte-area and local oscillations in the sample multimachine power system. However, in addition to be much simpler, JOA is a settings-free optimization method that does not require any algorithm-specific control parameters for its operation, which makes it more attractive for practical applications.

\section{APPENDIX}

A) Operating conditions

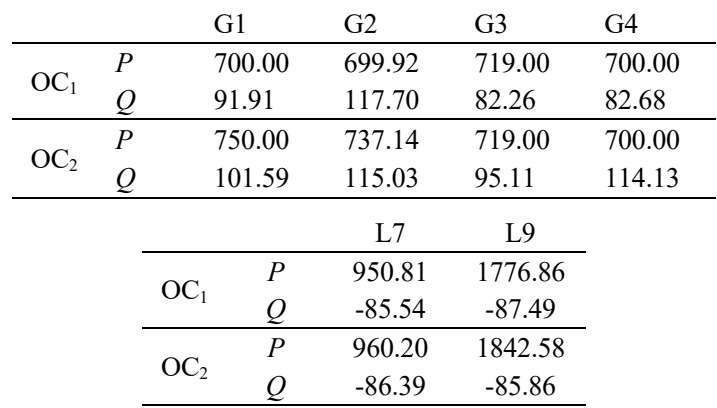

$P$ is given in MW and $Q$ in MVAR.
B) $\mathrm{MB}, \Delta \omega$ and $\Delta P_{a}$ PSS settings

MB-PSS: $F_{L}=0.2, F_{I}=1.25, F_{H}=12.0, K_{L}=30.0, K_{I}=40.0, K_{H}=160.0$,

$$
V_{\text {Lmax }}=0.075, V_{\text {Imax }}=V_{\text {Hmax }}=0.15
$$

$\Delta \omega$-PSS: $K=30.0, T_{1}=0.05, T_{2}=0.02, T_{3}=3.0, T_{4}=5.4$

$\triangle P_{a}$-PSS: $K=3.125, T_{1}=0.06, T_{2}=1.0$

Output limits for all PSSs are $[-0.15,0.15]$.

\section{ACKNOWLEDGMENT}

The authors acknowledge the Swiss National Science Foundation (SNSF) under the project number PZENP2_173628 of the program Ambizione Energy Grant (AEG). This research is also part of the activities of SCCER FURIES, which is financially supported by the Swiss Innovation Agency (Innosuisse-SCCER program).

\section{REFERENCES}

[1] J. Hossain and A. Mahmud, Large Scale Renewable Power Generation: Advances in Technologies for Generation, Transmission and Storage. New York: Springer, 2014.

[2] U. Hager, C. Rehtanz, and N. Voropai, Monitoring, Control and Protection of Interconnected Power Systems. New York: Springer, 2014.

[3] B. M. Buchholz and Styczynski, Smart Grids - Fundamentals and Technologies in Electricity Networks. Heidelberg: Springer, 2014.

[4] D. Saxena, S. N. Singh, and K. S. Verma, "Application of computational intelligence in emerging power systems," International Journal of Engeneering, Science and Technology, vol. 2, pp. 1-7, 2010.

[5] B. K. Panigrahi, A. Abraham, and S. Das, Computational Intelligence in Power Engineering. Berlin: Springer, 2010.

[6] A. P. Engelbrecht, Computational intelligence: an introduction, 2nd ed. West Sussex: John Wiley \& Sons Ltd., 2007.

[7] X. Yang, Nature-inspired optimization algorithms. MA: Elsevier, 2014.

[8] P. Kundur, Power System Stability and Control. New York: McGrawHill, 1994.

[9] M. Ramirez and O. P. Malik, "Simplified fuzzy logic controller and its application as a power system stabilizer," in Proc. IEEE International Conference on Intelligent System Applications to Power Systems, pp. 16, 2009.

[10] X-S. Yang, "A new metaheuristic bat-inspired algorithm," in Nature Inspired Cooperative Strategies for Optimization, Eds. J. R. Gonzalez et al. Berlin: Springer, 2010, pp. 65-74.

[11] R. Venkata-Rao, "Jaya: A simple and new optimization algorithm for solving constrained and unconstrained optimization problems," International Journal of Industrial Engineering Computations, vol. 7, pp. 19-34, 2016.

[12] R. Venkata-Rao, Jaya: An Advanced Optimization Algorithm and its Engineering Applications. Cham: Springer, 2019.

[13] Matlab $^{\circledR} /$ Simulink $^{\circledR}$ Software, MathWorks, R2019a.

[14] I. Kamwa, "Performance of three PSS for interarea oscillations." Retrieved July 1, 2020, from https://ch.mathworks.com/help/physmod/ sps/examples/performance-of-three-pss-for-interarea-oscillations.html

Miguel Ramirez-Gonzalez received his PhD Degree in Electrical Engineering from the University of Calgary, Canada, in 2008. From 1998 to 2020, he was working for the Mexican Institute for Electricity and Clean Energy. He is currently a Postdoctoral Research Associate at the Institute of Energy Systems and Fluid Engineering of the Zurich University of Applied Sciences. His research areas include modelling, simulation, analysis and control of power systems, large-scale wind/solar energy sources, HVDC links, FACTS devices, network support with battery energy storage, and the application of computational intelligence techniques to power system issues. 